\title{
Progranulin expression in neural stem cells and their differentiated cell lineages: An immunocytochemical study
}

\author{
LANHAI LÜ ${ }^{1 *}$, LI LUO ${ }^{1,2^{*}}$, YINGHONG LU ${ }^{2}$, LIZHI CHEN ${ }^{2}$, JIE XU $^{2}$ and KAIHUA GUO ${ }^{2}$ \\ ${ }^{1}$ Guanghua School of Stomatology, Hospital of Stomatology, Institute of Stomatological Research, \\ Guangdong Provincial Key Laboratory of Stomatology, Sun Yat-sen University, Guangzhou, \\ Guangdong 510055; ${ }^{2}$ Department of Anatomy and Neurobiology, Zhongshan School of \\ Medicine, Sun Yat-sen University, Guangzhou, Guangdong 510080, P.R. China
}

Received April 23, 2013; Accepted August 21, 2013

DOI: $10.3892 / \mathrm{mmr} .2013 .1664$

\begin{abstract}
Progranulin (PGRN) is a neurotrophic factor that regulates neurite outgrowth and enhances neuronal survival. The association between PGRN and neural stem cell (NSC) differentiation may aid in elucidating the underlying pathogenesis and potential treatments for neurodegenerative diseases. To investigate the association between PGRN and NSCs and their lineages, primary NSCs were prepared from the subventricular zone of neonatal Sprague Dawley pups (age, 1 day) and cultured in the neural stem cell medium. After 7 days in culture, NSCs aggregated into neurospheres and were maintained in the differential medium for 7 days following three passages. In addition, PGRN expression in neurospheres and differentiated cells (neurons, astrocytes and oligodendrocytes) were analyzed by immunocytochemistry. The PGRN expression and localization was also investigated in the brain tissues of neonatal rats (age, 1 and 7 days) by double immunofluorescence staining. The data indicated that PGRN was highly
\end{abstract}

Correspondence to: Dr Lanhai Lu, Guanghua School of Stomatology, Hospital of Stomatology, Institute of Stomatological Research, Guangdong Provincial Key Laboratory of Stomatology, Sun Yat-sen University, 56 Ling Yuan Road West, Guangzhou, Guangdong 510055, P.R. China

E-mail: 1vlanhai@mail.sysu.edu.cn

Dr Kaihua Guo, Department of Anatomy and Neurobiology, Zhongshan School of Medicine, Sun Yat-sen University, 74 Zhong Shan Er Road, Guangzhou, Guangdong 510080, P.R. China

E-mail: guokh@mail.sysu.edu.cn

*Contributed equally

Abbreviations: PGRN, progranulin; NSCs, neural stem cells; EGF, epidermal growth factor; bFGF, basic fibroblast growth factor; FTLD, frontotemporal lobar degeneration; DMEM, Dulbecco's modified Eagle's medium, FBS, fetal bovine serum; PBS, phosphatebuffered saline

Key words: progranulin, neural stem cells, cell differentiation, subventricular zone expressed in NSCs and their differentiated cell lineages in vitro. The results also demonstrated that PGRN was predominantly expressed in neurons and microglia in vivo, and marginally in NSCs, astrocytes and oligodendrocytes. The present study suggested that PGRN may be involved in the regulation of NSC differentiation.

\section{Introduction}

Progranulin (PGRN), also termed proepithelin, acrogranin or prostate cancer cell-derived growth factor, is a growth modulating factor involved in multiple biological functions (1). The mutations within the PGRN gene result in the partial loss of the PGRN protein, which is the leading cause of frontotemporal lobar degeneration (FTLD) (2). It was demonstrated that PGRN exhibited high expression levels during early neural development (3). However, its expression was restricted to defined neuronal populations, including cortical and hippocampal pyramidal neurons and Purkinje cells in the adult brain (4). Although the expression of PGRN in motor neurons and neuroblastoma cell lines has been investigated $(5,6)$, the involvement of PGRN in the central nervous system (CNS) remains to be elucidated.

Neural stem cells (NSCs) are the undifferentiated neural cells that possess multi-potential and self-renewal capacity, and differentiate into three cell lineages in the CNS: neurons, astrocytes and oligodendrocytes $(7,8)$. NSCs were predominantly separated and cultured from the subventricular zone (SVZ) of the lateral ventricles and subgranular zone (SGZ) of the hippocampus in the adult nervous system (9). Investigation of the involvement of ectogenic and endogenic factors in NSCs aid in the understanding of how to modulate the proliferation and differentiation of NSCs during neurogenesis $(10,11)$. Recent studies have demonstrated that PGRN is a neurotrophic factor that modulates neurite outgrowth and enhances neuronal survival (12), and exogenous PGRN promotes neural progenitor cell proliferation (13). However, the expression profiles of PGRN in neurospheres and its differentiated subpopulations are not fully understood.

Considering the essential role of NSCs in neurogenesis, further insight into the association between PGRN and NSCs may aid in the clarification of the involvement of PGRN in 
the pathogenesis of neurodegenerative diseases and provide a basis for future novel therapies. In the present study, primary NSCs were isolated and cultured from the neonatal rat brain and were used to investigate the expression of PGRN in NSCs and differentiated subpopulations.

\section{Materials and methods}

Primary culture of rat neural stem cells. All animal related experiments were conducted in accordance with the guidelines of the Experimental Laboratory Animal Committee of Sun Yat-sen University Health Science Center (Guangzhou, China) and under the approval of the principles of the National Institutes of Health's Guide for the Care and Use of Laboratory Animals. NSCs were prepared from the SVZ of the brain of neonatal Sprague Dawley (SD) rats. Neonatal SD pups (age, 1 day) were sacrificed and their brains were removed. The meninges and blood vessels were removed with fine forceps; a thin tissue layer surrounding the lateral ventricles was isolated from coronal slices of the brain and cut into small sections in ice-cold D-Hanks medium (Genom Co., Hangzhou, China) containing $100 \mathrm{U} / \mathrm{ml}$ penicillin and $100 \mathrm{mg} / \mathrm{ml}$ streptomycin. Following two washes in D-Hanks medium, the tissues were dissociated mechanically with a Pasteur pipette (Biolegix, Philadelphia, PA, USA) into coarse single-cell suspensions, which were further centrifuged and resuspended prior to being passed through a cell strainer $\left(40-\mu \mathrm{m}, 352340\right.$, BD Falcon ${ }^{\mathrm{TM}}$; BD Biosciences, Franklin Lakes, NJ, USA) to remove the debris. When the homogenate was centrifuged at $80 \mathrm{xg}$ for $5 \mathrm{~min}$, the cells were resuspended in supplemented culture medium that consisted of DMEM: nutrient mixture F12 (DMEM/F12) (1:1) (Gibco-BRL, Carlsbad, CA, USA) supplemented with 1\% B27 (Invitrogen Life Technologies, Carlsbad, CA, USA), 1\% N2 supplement (17502-048; Invitrogen Life Technologies), $20 \mathrm{ng} / \mathrm{ml}$ epidermal growth factor (EGF) (Invitrogen Life Technologies) and $20 \mathrm{ng} / \mathrm{ml}$ basic fibroblast growth factor (bFGF) (Invitrogen Life Technologies). Cells were incubated at $37^{\circ} \mathrm{C}$ in a $5 \% \mathrm{CO}_{2}$ atmosphere and half the volume of the culture medium was replaced every 2 days. After 7 days, NSCs aggregated into neurospheres. For passaging, neurospheres were resuspended and replated into non-coated culture flasks at a density of $2 \times 10^{5}$ cells $/ \mathrm{ml}$ following mechanical dissociation.

Differentiation of neural stem cells. To induce the differentiation of NSCs, neurospheres were seeded onto poly-L-lysine-coated glass coverslips (NEST Biotechnology Co., Ltd., Shanghai, China) following three passages and then cultured in differentiation medium containing DMEM/F12 (1:1), $1 \%$ N2, and 10\% fetal bovine serum (FBS) (Gibco-BRL). Under this environment, the sedimentary neurospheres attached to the poly-L-lysine-coated surface and began to differentiate into neurons, astrocytes and oligodendrocytes. The cells were maintained at $37^{\circ} \mathrm{C}$ in a $5 \% \mathrm{CO}_{2}$ atmosphere and the medium was replaced every 2 days. The differentiation of NSCs was observed using a phase-contrast microscope (DMI4000B; Leica, Wetzlar, Germany), cells were photographed and analyzed for morphology at day 7 .

Preparation of tissue sections. The brains of neonatal SD rats (age, 1 and 7 days) were used to investigate the expression and localization of PGRN during development. The rats were anesthetized with a peritoneal injection of $10 \%$ chloral hydrate (Sigma-Aldrich, St. Louis, MO, USA) and perfused transcardially with cold phosphate saline buffer (PBS) followed by ice-cold $4 \%$ paraformaldehyde in $0.1 \mathrm{M}$ PBS. The brains were removed and postfixed in $4 \%$ paraformaldehyde solution at $4^{\circ} \mathrm{C}$ overnight, after which the brains were dehydrated serially in 10,20 and $30 \%$ sucrose in PBS overnight at $4^{\circ} \mathrm{C}$. When the the brain sediment reached the bottom of the tubes, the brains were embedded in Optimal Cutting Temperature compound (Sakura Finetek Co., Ltd., Tokyo, Japan). Serial coronal sections were cut using a freezing microtome at $10 \mu \mathrm{m}$ and mounted onto poly-L-lysine-coated glass slides. PGRN was detected using immunohistochemistry.

Immunocytochemistry staining. To investigate the expression of PGRN in NSCs, the neurospheres were collected by centrifugation and then washed three times with PBS and fixed in $4 \%$ paraformaldehyde solution for $30 \mathrm{~min}$ at room temperature. Following three washes with PBS, the neurospheres were blocked using $10 \%$ normal goat serum for $1 \mathrm{~h}$ at room temperature to reduce non-specific binding. Neurospheres were then incubated with primary antibody against nestin (dilution, 1:1,000; Abcam, Hong Kong, China) for NSCs and PGRN (dilution, 1:500; R\&D Systems, Inc., Minneapolis, MN, USA) in PBS containing 0.3\% Triton X-100 at $4^{\circ} \mathrm{C}$ overnight. Following sufficient washing, cells were incubated with appropriate fluorescence-conjugated secondary antibodies for $1 \mathrm{~h}$ at $37^{\circ} \mathrm{C}$. For the identification of PGRN in differentiated NSCs, the cells were grown on poly-L-lysine-coated round glass coverslips, rinsed three times with PBS following 7 days of differentiation, fixed with $4 \%$ paraformaldehyde solution for $30 \mathrm{~min}$ at room temperature and blocked with $10 \%$ normal goat serum for $1 \mathrm{~h}$ at room temperature. The cells were then incubated with primary antibody against $\beta$ III-tubulin for neurons (dilution, 1:1,000; Abcam), glial fibrillary acidic protein (GFAP) for astrocytes (dilution, 1:1,000; Abcam), Oli for oligodendrocytes (dilution, 1:1,000; Abcam) and PGRN (dilution, 1:500; R\&D Systems, Inc.) in PBS containing 0.3\% Triton X-100 at $4^{\circ} \mathrm{C}$ overnight. The cells were washed with PBS and incubated with the corresponding fluorescence-conjugated secondary antibodies [Alexa Fluor ${ }^{\circledR} 488$ donkey anti-sheep IgG (H+L), A-11015; Alexa Fluor ${ }^{\circledR} 594$ goat anti-mouse IgG $(\mathrm{H}+\mathrm{L}), \mathrm{A}-11005$; and Alexa Fluor ${ }^{\circledR} 594$ goat anti-rabbit IgG (H+L), A-11012; Invitrogen Life Technologies)] for $1 \mathrm{~h}$ at $37^{\circ} \mathrm{C}$. Nuclei were stained for $5 \mathrm{~min}$ with the nuclear dye Hoechst 33342 (20 ng/ml; Biotium, Hayward, CA, USA). Images were captured with a fluorescence microscope (BX51WI; Olympus, Tokyo, Japan).

Double immunofluorescent staining. The expression of PGRN was also detected in the brain of neonatal SD rats (age, 1 and 7 days). Frozen sections were heated to $56^{\circ} \mathrm{C}$ for $10 \mathrm{~min}$, washed with PBS and incubated with blocking solution with $10 \%$ normal goat serum containing $0.3 \%$ Triton X-100 for $1 \mathrm{~h}$ at room temperature. The sections were then incubated with primary antibodies (anti-PGRN, 1:600; anti- $\beta$ III-Tubulin, 1:1,000; anti-GFAP, 1:1,000; and anti-Oli, 1:1,000) overnight at $4^{\circ} \mathrm{C}$. When the sections had been thoroughly washed with PBS, 
A

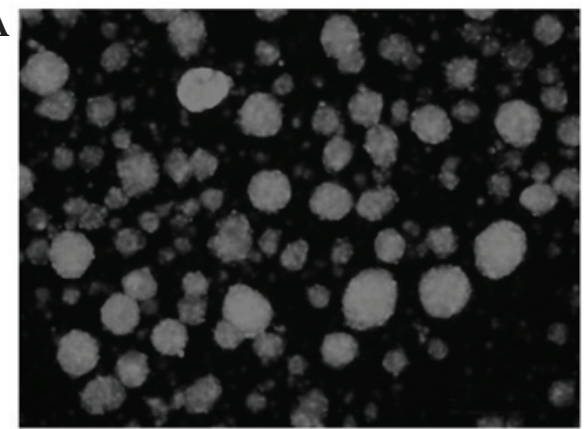

B

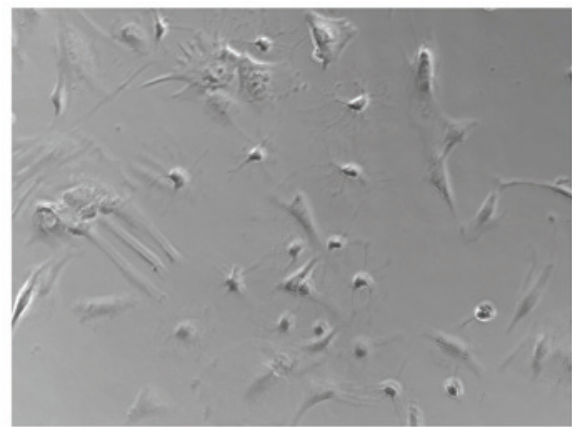

C
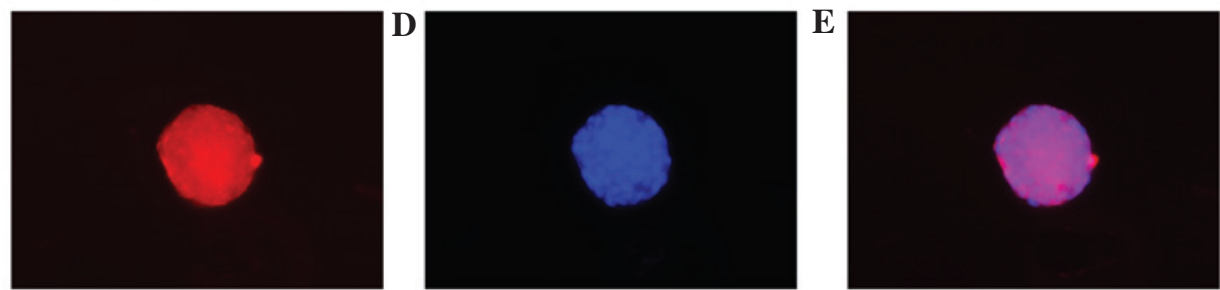

$\mathbf{F}$
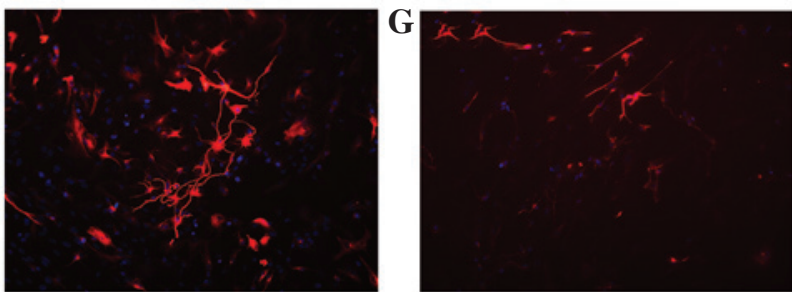

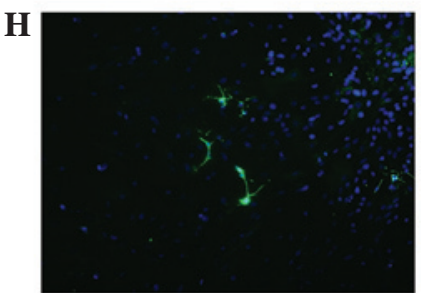

Figure 1. (A,C-E) Characteristics of neurospheres isolated and cultured from the subventricular zone of neonatal SD rats, (magnification, x100). Immunocytochemical staining showed that neurospheres appeared positive when tested for nestin, an intermediate filament characteristic of undifferentiated neuroepithelial cells. (A) Neurospheres maintained in the culture medium for 7 days. (B) When neurospheres were maintained in the differential medium for 7 days, the neurosphere-derived cells grow onto the coverslips and gradually showed the morphological features of neural cells. (C) Nestin (red) expression was observed in the neurospheres in primary cultures. (D and H) Cell nuclei were staining with Hoechst 33342 (blue). (B,F-H) Neural stem cells (NSCs) differentiate into neurons, astrocytes and oligodendrocytes, (magnification, x200). The immunocytochemical staining shows that the NSCs differentiate into (F) $\beta$ III-tubulin ${ }^{+}$neurons, (G) glial fibrillary acidic protein ${ }^{+}$astrocytes and (H) $\mathrm{Oli}^{+}$oligodendrocytes .

appropriate secondary antibodies Alexa Flour 488 and Alexa Flour 594 (1:1,000 dilution, Invitrogen Life Technologies) were added and incubated for $2 \mathrm{~h}$ at $37^{\circ} \mathrm{C}$, and the sections were washed three times with PBS. Nuclei were stained with the nuclear dye Hoechst $33342(20 \mathrm{ng} / \mathrm{ml})$ for $5 \mathrm{~min}$ and rinsed with PBS, and the coverslips were mounted on slides with FluorSave reagent (Beyotime, Jiangsu, China).

\section{Results}

Neural stem cells aggregate into neurospheres and express $P G R N$. Neurospheres were clearly observed at day 3. Numerous neurospheres proliferated in suspension at day 7 following primary culture (Fig. 1A). Immunocytochemistry results showed that neurospheres appeared positive for nestin and PGRN expression (Fig. 2A-D). These results indicated that NSCs were successfully isolated and cultured and expressed PGRN.

NSC-derived subpopulations express PGRN. After three passages, the neurospheres were transferred in 24-well plates containing poly-L-lysine-coated glass coverslips and were maintained in differential medium, containing DMEM/F12 (1:1), $1 \%$ N2 supplement and 10\% FBS, for a further 7 days. The neurospheres began to attach to the coverslips and
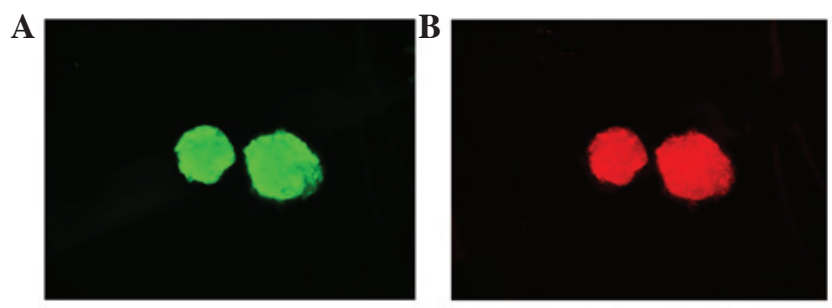

C
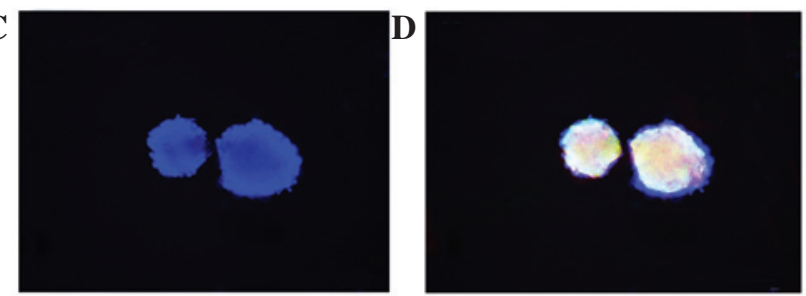

Figure 2. Co-expression of progranulin (PGRN) and nestin in neural stem cells (NSCs) in vitro. Neurospheres derived from the subventricular zone of neonatal Sprague Dawley rats maintained in the NSC culture medium for 7 days were stained for (A) PGRN (green), (B) nestin (red) and (C) Hoechst 33342 (blue). (D) PGRN co-expressed with nestin in neurospheres Magnification, x100.

gradually showed the morphological features of neural cells (Fig. 1B). The immunocytochemical staining showed that the NSCs differentiated into $\beta$ III-tubulin ${ }^{+}$neurons, GFAP ${ }^{+}$ 


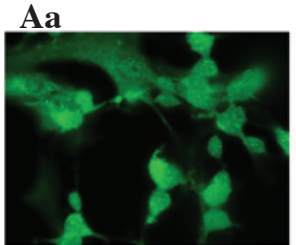

Ba

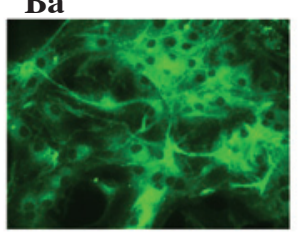

$\mathrm{Ca}$

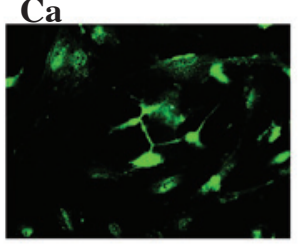

$\mathrm{Ab}$

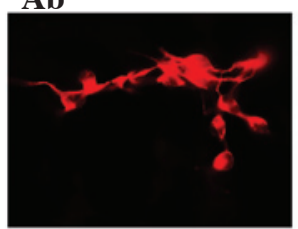

$\mathrm{Bb}$

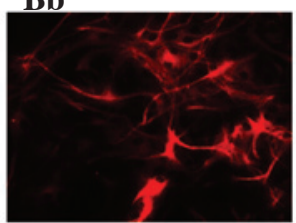

$\mathrm{Cb}$

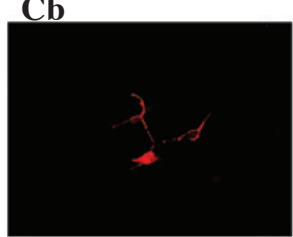

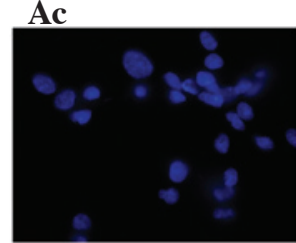
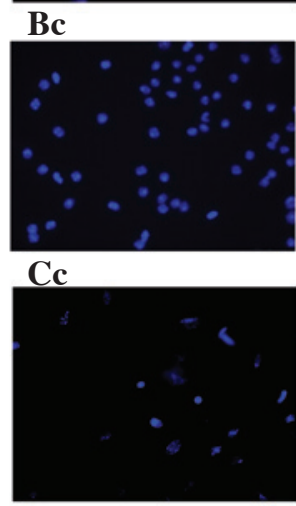

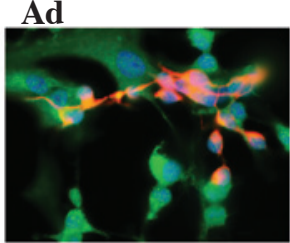

Bd
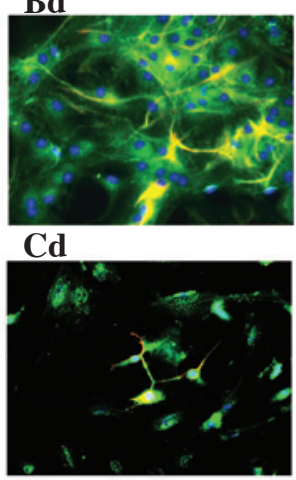

Figure 3. Differentiated neural stem cells (NSCs) express progranulin (PGRN) in vitro. PGRN colocalizes with neuron, astrocyte and oligodendrocyte markers (Aa-Ad) PGRN (green) expression in $\beta$ III-tubulin ${ }^{+}$neurons (red, magnification, x1000). (Ba-Bd) PGRN expression in glial fibrillary acidic protein ${ }^{+}$astrocytes (red; magnification, x400). (Ca-Cd) PGRN expression in Oli ${ }^{+}$oligodendrocytes (red, magnification, $\mathrm{x} 400$ ).
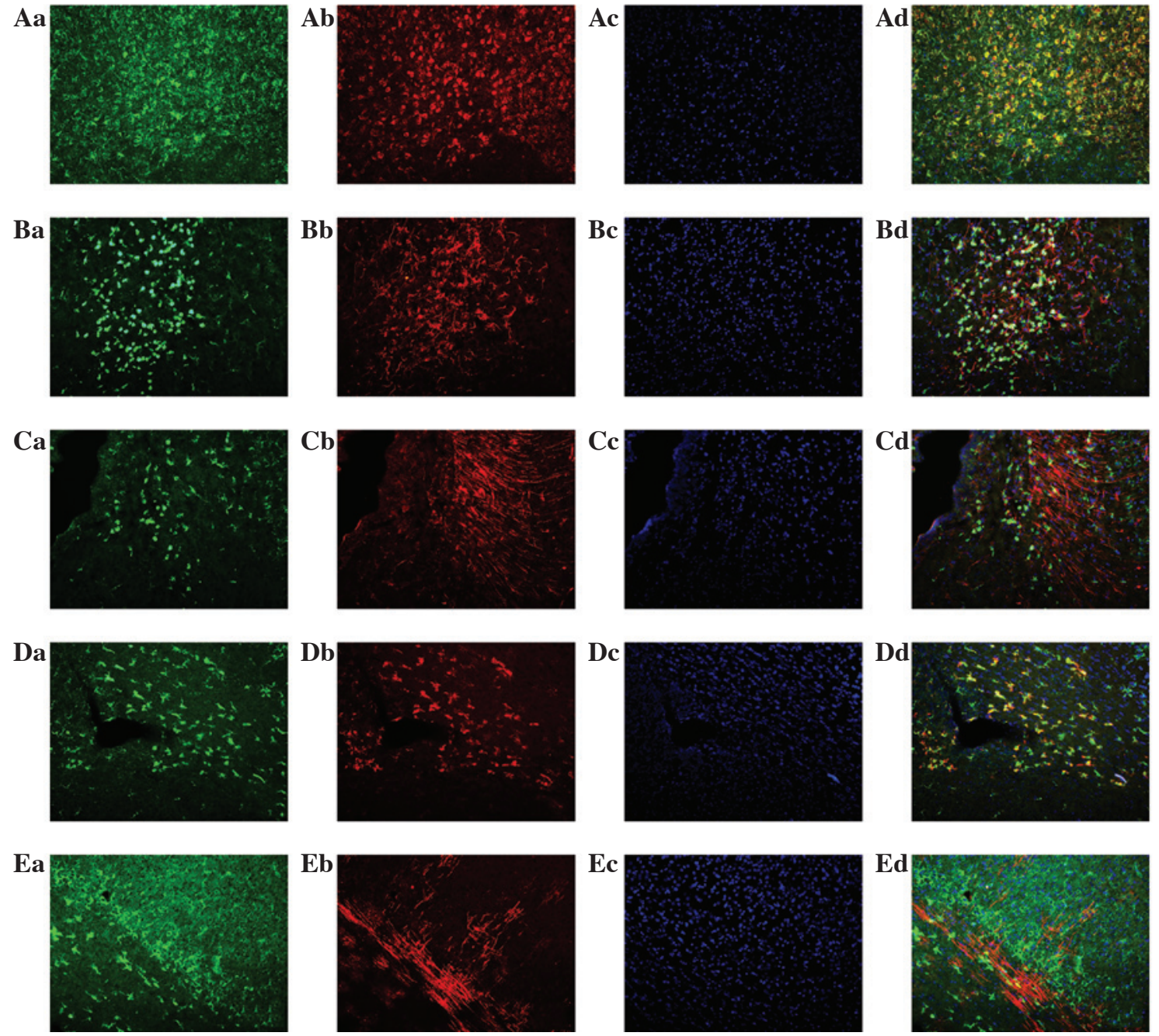

Figure 4. Progranulin (PGRN) is expressed predominantly in neurons and microglia in vivo, and marginally in neural stem cells (NSCs), astrocytes and oligodendrocytes in the brains of neonatal rats. PGRN (green) is expressed in Tubulin ${ }^{+}$neurons (red) (Aa-Ad) and OX-42+ microglia (Da-Dd). The immunofluorescence results also showed a lack of expression of PGRN in nestin ${ }^{+}$NSCs (red) $(\mathrm{Ca}-\mathrm{Cd})$, GFAP ${ }^{+}$astrocytes (red) (Ba-Da) and Oli ${ }^{+}$oligodendrocytes (red) (Ea-Ed). Images Aa-Ad and Da-Dd showed that PGRN was co-expressed with $\beta$ III-tubulin in neurons and OX-42+ in microglia of the neonatal rats (magnification, $\mathrm{x} 200$ ). 
astrocytes and $\mathrm{Oli}^{+}$oligodendrocytes (Fig. 1F-H). Moreover, all NSC-derived cells expressed PGRN in vitro (Fig. 3).

\section{PGRN is}

expressed in neurons and microglia but marginally in NSCs, astrocytes and oligodendrocytes in the brains of neonatal rats. The expression of PGRN was also analyzed by immunofluorescent staining in postnatal rat brains at day 1 (P1) and day 7 (P7). PGRN immunoreactivity was observed predominantly in the cortex, corpus callosum and hippocampus. To analyze the cell type-specific expression of PGRN in the neonatal brain, immunofluorescent double labeling was performed with specific markers for neurons ( $\beta$ III-tubulin), astrocytes (GFAP), microglia (OX-42), NSCs (Nestin) and oligodendrocytes (Oli). The results indicated that the mature neuronal marker, $\beta$ III-tubulin (Fig. 4Aa-Ad) and microglia marker, OX-42 (Fig. 4Da-Dd) were double-labeled with PGRN, which indicated that PGRN was predominantly expressed in neurons and microglia during the early stages of rat brain development. By contrast, PGRN immunoreactivity was marginal in astrocytes (Fig. 4Ab-Db), NSCs (Fig. 4Ca-Cd) and oligodendrocytes (Fig. 4Ea-Ed). Thus, these results suggest that PGRN was predominantly expressed in neurons and microglia rather than NSCs, astrocytes and oligodendrocytes in the brain at P1 and P7.

\section{Discussion}

Numerous studies have demonstrated that the $68.5-\mathrm{kDa}$ secreted protein PGRN is a growth factor (14), which functions as a potential mediator of wound healing and cancer progression (1). More recently, PGRN was suggested to be associated with neurodegenerative diseases and psychiosis $(15,16)$. Mutations in the progranulin gene have been identified as a predominant cause of frontotemporal lobar degeneration with ubiquitin-positive inclusions (17). Previous studies have indicated that the overexpression of PGRN was cytoprotective in cultured cells (5) and in vivo in transgenic mice (18). PGRN was observed to be an endogenous neuroprotectant against apoptosis and inflammatory responses. Conversely, extracellular PGRN also acts as a neuroprotective factor in the regulation of neurite outgrowth, enhanced neuronal survival and protection against excitotoxicity and oxidative stress which leads to the exacerbation of neuronal cell death $(12,19)$. Taken together, these results demonstrated that PGRN may be a therapeutic target for neurodegenerative diseases.

It was demonstrated that PGRN also enhances neural progenitor cell proliferation (13). In the present study, the expression of PGRN was analyzed in NSCs cultured from the SVZ of neonatal rat brain and their differentiated cell lineages. The expression and cell location of PGRN was also detected in the neonatal rat brain. The results indicated that PGRN was abundantly expressed in neurospheres and their differentiated cell lineages. The in vivo studies showed that PGRN was highly expressed in neurons and microglia but only marginally in NSCs, astrocytes and oligodendrocytes in neonatal rat brain. These data provided useful information concerning the involvement of PGRN in the regulation of NSCs under the physiological conditions during development.
PGRN was identified in motor neurons, neuroblastoma cell lines and fibroblasts $(5,20,21)$. Our data indicated that PGRN may be important in regulating the differentiation of NSCs under physiological conditions during development. In the present study, the expression of PGRN was analyzed in NSCs in vitro, and the results showed that PGRN was co-expressed with nestin in the NSC cytosol during proliferation. The immunostaining for PGRN by different neural cell markers nestin, $\beta$ III-Tubulin, GFAP and Oli was also used to analyze the in vitro NSC differentiation. The in vivo studies suggested that PGRN was co-expressed with neuron and microglia markers, but not with markers of NSCs, astrocytes and oligodendrocytes. The differential PGRN expression between in vitro and in vivo studies may be due to the different environmental stimulus. The data also demonstrated the expression of PGRN in neurons increased during the cell maturation, whereas the expression level in microglia was dependent upon the activation state of the cells, as it was upregulated in microglia in response to excitotoxic injuries (22). Astrocytes differentiated from in vitro NSCs exhibited a high level of PGRN, indicating that the astrocytes are a potential source for cell transplantation during the treatment of FTLD.

In conclusion, the present data suggest that PGRN is likely to be involved in regulating the differentiation of NSCs. PGRN expression may be altered following oxidative stress or other environmental stimuli. Therefore, further studies should focus on the mechanism underlying the regulation of the differentiation and maturation of NSCs and its subpopulations by PGRN during development.

\section{Acknowledgements}

This study was supported by the National Natural Science Foundation of China for Youth (grant no. 81000562), the National innovation experiment program for University students (grant no. 101055827) and the Medical Scientific Research Foundation of Guangdong Province, China (grant no. B2010068).

\section{References}

1. He Z and Bateman A: Progranulin (granulin-epithelin precursor, P-cell-derived growth factor, acrogranin) mediates tissue repair and tumorigenesis. J Mol Med (Berl) 10: 600-612, 2003.

2. Gass J, Lee WC, Cook C, et al: Progranulin regulates neuronal outgrowth independent of sortilin. Mol Neurodegener 7: 33, 2012.

3. Daniel R, He Z, Carmichael KP, Halper J and Bateman A: Cellular localization of gene expression for progranulin. J Histochem Cytochem 48: 999-1009, 2000.

4. Daniel R, Daniels E, He Z and Bateman A: Progranulin (acrogranin/PC cell-derived growth factor/granulin-epithelin precursor) is expressed in the placenta, epidermis, microvasculature, and brain during murine development. Dev Dyn 227: 593-599, 2003.

5. Ryan CL, Baranowski DC, Chitramuthu BP, Malik S, Li Z, Cao M, Minotti S, Durham HD, Kay DG, Shaw CA, Bennett HP and Bateman A: Progranulin is expressed within motor neurons and promotes neuronal cell survival. BMC Neurosci 10: 130, 2009.

6. Piscopo P, Rivabene R, Adduci A, Mallozzi C, MalvezziCampeggi L, Crestini A and Confaloni A: Hypoxia induces up-regulation of progranulin in neuroblastoma cell lines. Neurochem Int 57: 893-898, 2010.

7. Yanagisawa M and Yu RK: The expression and functions of glycoconjugates in neural stem cells. Glycobiology 17: 57R-74R, 2007. 
8. Park JH, Choi MR, Park KS, Kim SH, Jung KH and Chai YG: The characterization of gene expression during mouse neural stem cell differentiation in vitro. Neurosci Lett 506: 50-54, 2012.

9. Bergström T and Forsberg-Nilsson K: Neural stem cells: brain building blocks and beyond. Ups J Med Sci 117: 132-142, 2012.

10. Trujillo CA, Schwindt TT, Martins AH, Alves JM, Mello LE and Ulrich $\mathrm{H}$ : Novel perspectives of neural stem cell differentiation: from neurotransmitters to therapeutics. Cytometry A 75: 38-53, 2009.

11. Alvarez-Buylla A and Lim DA: For the long run: maintaining germinal niches in the adult brain. Neuron 41: 683-686, 2004.

12. Van Damme P, Van Hoecke A, Lambrechts D, Vanacker P, Bogaert E, van Swieten J, Carmeliet P, Van Den Bosch L and Robberecht W: Progranulin functions as a neurotrophic factor to regulate neurite outgrowth and enhance neuronal survival. J Cell Biol 181: 37-41, 2008.

13. Nedachi T, Kawai T, Matsuwaki T, Yamanouchi $\mathrm{K}$ and Nishihara M: Progranulin enhances neural progenitor cell proliferation through glycogen synthase kinase $3 \beta$ phosphorylation. Neuroscience 185: 106-115, 2011.

14. Bateman A and Bennett HP: Granulins: the structure and function of an emerging family of growth factors. J Endocrinol 158: $145-151,1998$

15. De Muynck L and Van Damme P: Cellular effects of progranulin in health and disease. J Mol Neurosci 45: 549-560, 2011.
16. Galimberti D, Dell'Osso B, Fenoglio C, et al: Progranulin gene variability and plasma levels in bipolar disorder and schizophrenia. PLoS One 7: e32164, 2012.

17. Gass J, Prudencio M, Stetler C and Petrucelli L: Progranulin: an emerging target for FTLD therapies. Brain Res 1462: 118-128, 2012.

18. Tao J, Ji F, Wang F, Liu B and Zhu Y: Neuroprotective effects of progranulin in ischemic mice. Brain Res 1436: 130-136, 2012.

19. Xu J, Xilouri M, Bruban J, Shioi J, Shao Z, Papazoglou I, Vekrellis K and Robakis NK: Extracellular progranulin protects cortical neurons from toxic insults by activating survival signaling. Neurobiol Aging 32: 2326, 2011.

20. Guerra RR, Kriazhev L, Hernandez-Blazquez FJ and Bateman A Progranulin is a stress-response factor in fibroblasts subjected to hypoxia and acidosis. Growth Factors 25: 280-285, 2007.

21. Naphade SB, Kigerl KA, Jakeman LB, Kostyk SK, Popovich PG and Kuret J: Progranulin expression is upregulated after spinal contusion in mice. Acta Neuropathol 119: 123-133, 2010.

22. Petkau TL, Neal SJ, Orban PC, MacDonald JL, Hill AM, Lu G, Feldman HH, Mackenzie IR and Leavitt BR: Progranulin expression in the developing and adult murine brain. J Comp Neurol 518: 3931-3947, 2010. 